\title{
Development of a System for Proactive Information Service
}

\author{
Myon-Woong Park ${ }^{1}$, Soo-Hong Lee ${ }^{2}$, Young-Tae Sohn ${ }^{1}$, Jae Kwan Kim ${ }^{1}$, \\ Ilju Bae', and Jae-Kwon $\mathrm{Lim}^{2}$, \\ ${ }^{1}$ Intelligent and Interaction Research Center, Korea Institute of Science and Technology, \\ P.O. BOX 131, Cheongryang, Seoul, Korea \\ \{myon, ytsohn, kimjk, iljusado\}@kist.re.kr \\ ${ }^{2}$ Dept. Of Mechanical Engineering, Yonsei University, \\ 50, Yonsei-ro, Seodaemun-gu, Seoul, Korea \\ \{shlee, 1jk1225\} @yonsei.ac.kr
}

\begin{abstract}
This paper describes a Context-based Information Service Agent which has been developed in the domain of sports, especially for baseball game which often called a sport based on data. For the implementation of the agent, a knowledge model structure which can define the change of context along the axis of time has been suggested. The usability of the agent has been proved as the service system implemented on mobile phone proactively recommended the observation points and information suitable for the current context of watching the game in non-invasive manner.
\end{abstract}

Keywords: information service, context-awareness, agent, knowledge model, mobile platform.

\section{Introduction}

Rapid and remarkable progress in computing environment such as world wide web and powerful yet affordable server enable the application programs to provide humans with more intelligent and proactive information service. Software agent is usually the kernel of the service, and intelligent agents for information service in many different domains such as finance, traffic, e-commerce, etc. have been developed for satisfying user's requirement and providing practical service [1, 2, 3, 4]. Among those agents, the context based information service agent is supposed to recognize current situation or change of situation of information user in order to supply information most useful for the user at the context. Recognition of the situation is based on the overall assessment about all the related factors. For the representation of situation, the change over time should be considered as one of the important factors since the agent needs to adapt to dynamically changing situation and correct its behavior [5]. Most of the existing agent are not that much adaptive to the change over time, though.

The method of knowledge modeling is necessary for expanding the range of representation of situation in order to reflect the change of situation over time. While this paper introduces a case of the implementation of a context aware information agent, a structure of the ontology as a knowledge model which can define the change of situation over time is suggested. 
Baseball game continues couple of hours and the situation consisted of many factors like pitcher, batter, runner, inning, out count, ball count, etc. keep changing. At every change, game audience might be interested in specific data through which the progress of the game is predicted. For instance, the record on the stolen base of a runner might be more interesting than the hitting average of a batter if the runner is on the first base with out-count one at late inning while both team scored nil. The possibility of stealing base at the circumstance is usually reminded and the record of the runner is provided by commentator in case of broadcasting. There can be many 'observation points' such as the possibility of stealing base during the baseball game watch. The above mentioned agent was regarded as the kernel of artifact being capable of playing the role of commentator and recommending proactively the observation points suitable for the game context. The agent has been implemented on a smart phone, which becomes the powerful platform for mobile internet and the most useful personal assistant.

\section{Context Based Information Service Agent}

As the agent needs to recognize current situation and recommend information appropriate for the context, it requires number of functionalities such as context-awareness, inference, information retrieval, and priority indexing (Fig. 1) [6].



Fig. 1. Functions required for the intended Information Service

For the context awareness, the context modeling technology for the formalization of the recognized context data is necessary. The context recognized through context sensing technology is represented in a formalized model and language. The function to recognize and assess current context by analyzing the model is identified as context recognition. Clear definition on the context elements is necessary for context awareness. And also, grouping of the elements and converting them into knowledge are necessary. Once current context is recognized, the agent refers to the knowledge model and infers the information appropriate for the context. The knowledge model defines context element and information element for generating the relation between two elements in knowledge map. Then, the agent retrieves the specific information relevant to the context from the database and supplies it to the user. 


\section{Information Service for Watching Baseball Game}

There are various records in baseball game as is often referred as the game of data. They are usually provided by caster or commentator. But, inevitably the service is unidirectional and limited in quality and quantity in many cases. Introduction of the 'observation point' and providing the observation point and relevant statistics in suitable timing proactively by the system would improve the service and add the zest to watching the game. The observation point means the group of the player record relevant to the current context, which attracts audience's interest. The system structure of the contextbased information agent applied to the baseball game watch is depicted in Fig. 2. The role of each module on the structure diagram is to be introduced, and the flow from context recognition to providing observation points is explained in this section.

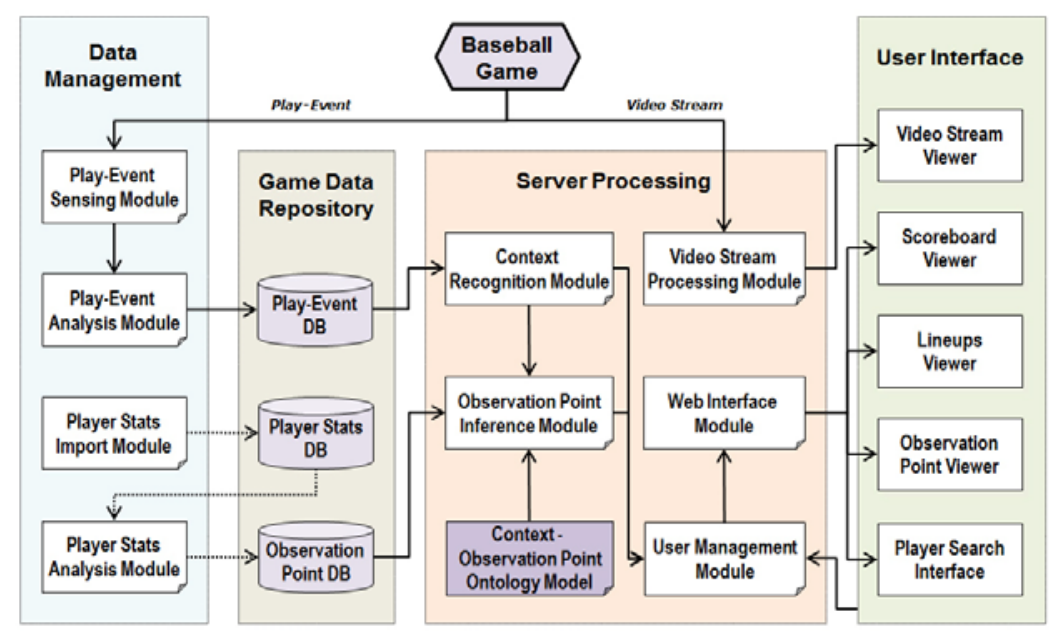

Fig. 2. System Structure of the context-based information agent

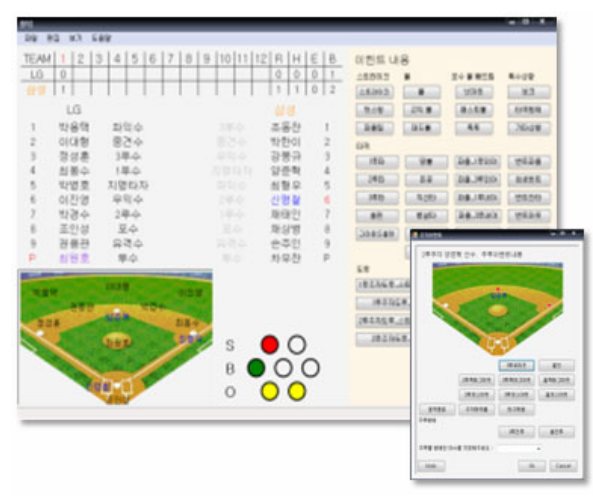

Fig. 3. Record Interface

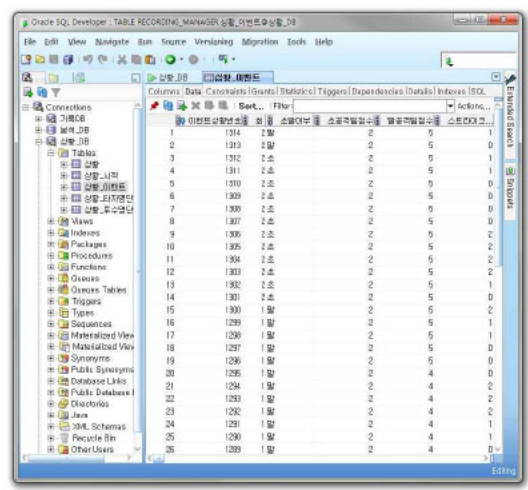

Fig. 4. Context Model 
Provisioning of observation points starts when an event occurs, which mean the change of the situation. The event is recorded through the interface as shown in Fig. 3 and analyzed for creating the context model which is then stored in DB as shown in Fig 4. The occurrence of an event is started at the moment of ball drawing by pitcher in this work.

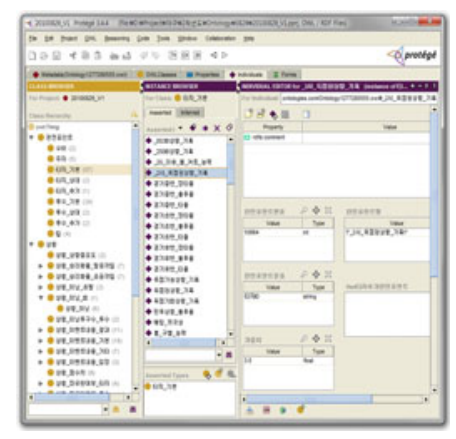

Fig. 5. Modeling of the Relation between Context and Observation Point using Protégé

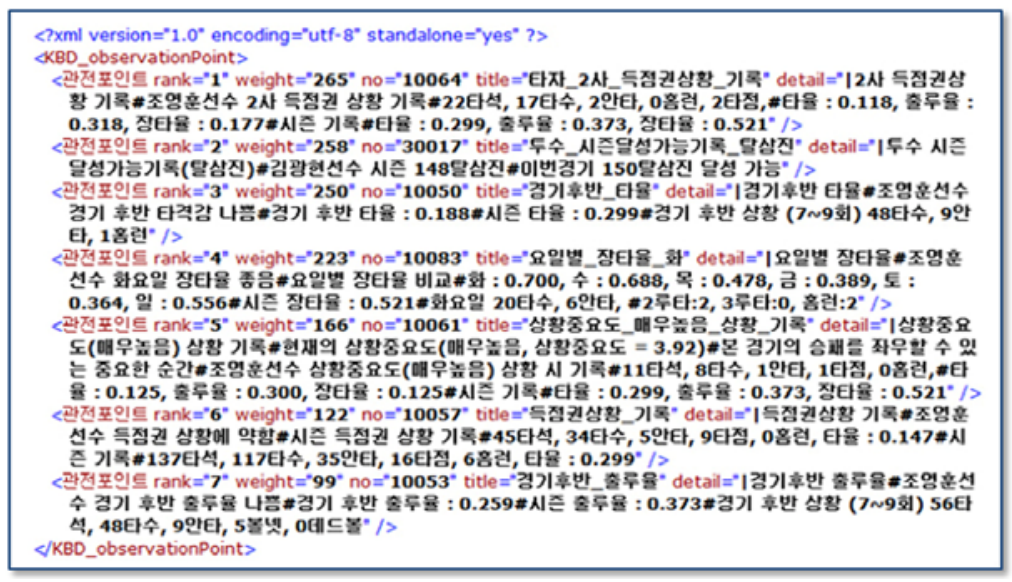

Fig. 6. Observation Points converted to XML file

The server regularly checks the change in context DB. In case of any change detected, the server identifies the context and extracts observation points relevant to the context. Then, the observation points are served through the user interface. The extraction of the observation points are carried out through the ontology model for the relation between context and observation point. The server compares the current context and the ones defined in the ontology model by parsing the relation model in order to extract the matching context instance. Then, the observation points having the relation with the extracted context are derived. The matching context instance is extracted and the observation point instances having the relation with the extracted context are inferred. The observation point instance includes name of the related table, 
name of column, searching constraints, etc., with which matching observation points stored in the analysis DB are extracted at the server (Fig. 5). The observation point implies the meaningful data of the players resulted from the analysis on the record $\mathrm{DB}$, which is stored in analysis DB. Weight value is given to each instance at the ontology model in order for comparing the importance of the observation point. The selected observation points adequate for the current context are listed in the order of weight values given to the observation points.

The derived observation points are changed into XML files as shown on Fig. 6 at the web server and sent to UI through http request communication. Through this integrated process, the agent proactively provides human users with context relevant observation points, and the users are able to get interesting record information.

\section{Knowledge Model}

The structure of the ontology model for the representation of the relation between context and observation point is shown in Fig. 7. Ontology is a knowledge model represented in the form which can be handled by computer. As the definition of all the terms and their relationship are logically described in otology, the model enables formal representation and declarative description of knowledge. Ontology can be applied in many different domains for establishing knowledge base as knowledge management and expansion and inference are possible through ontology.

\begin{tabular}{|c|c|c|c|c|c|c|c|}
\hline \multicolumn{3}{|c|}{ Previous Context } & \multicolumn{3}{|c|}{ Context } & \multicolumn{2}{|c|}{ Observation Point } \\
\hline PK & \multicolumn{2}{|c|}{ Instance Name of Previous Context } & PK & \multicolumn{2}{|c|}{ Instance Name of Context } & PK & Instance Name of Observation Point \\
\hline \multirow{26}{*}{ DP } & \multicolumn{2}{|c|}{ Importance of Context } & \multirow{25}{*}{ DP } & \multicolumn{2}{|c|}{ Importance of Context } & \multirow{5}{*}{ DP } & Name of Observation Point \\
\hline & \multirow{2}{*}{$\begin{array}{l}\text { Winning } \\
\text { probability }\end{array}$} & Top Team & & \multirow{2}{*}{$\begin{array}{l}\text { Winning } \\
\text { probability }\end{array}$} & Top Team & & Classification of Observation Point \\
\hline & & Bottom Team & & & Bottom Team & & Observation Point No. \\
\hline & \multirow{2}{*}{ Inning } & Inning Number & & \multirow{2}{*}{ Inning } & Inning Number & & Necessary Data \\
\hline & & Top/Bottom & & & Top/Bottom & & Weight Value \\
\hline & \multirow{4}{*}{ Event Contents } & Basic & & \multirow{4}{*}{ Event Contents } & Basic & OP & Has Additional Obs ervation Point \\
\hline & & Result & & & Result & \multirow{3}{*}{\multicolumn{2}{|c|}{ Additional Observation Point }} \\
\hline & & Position & & & \begin{tabular}{|l|} 
Position \\
\end{tabular} & & \\
\hline & & etc & & & etc & & \\
\hline & \multicolumn{2}{|l|}{ Score Difference } & & \multicolumn{2}{|l|}{ Score Difference } & \multirow[b]{2}{*}{ PK } & Instance Name of Additional \\
\hline & \multirow{2}{*}{$\begin{array}{l}\text { Left/Right } \\
\text { Handed Pitcher }\end{array}$} & Hitter & & \multirow{2}{*}{$\begin{array}{l}\text { Left/Right } \\
\text { Handed Pitcher }\end{array}$} & Hitter & & Observation Point \\
\hline & & Pitcher & & & Pitcher & \multirow{5}{*}{ DP } & Name of Observation Point \\
\hline & \multirow{4}{*}{ Runner } & 15: Base & & \multirow{4}{*}{ Runner } & 15: Base & & Classification of Observation Point \\
\hline & & $2^{\text {nd }}$ Base & & & $2^{\text {nd }}$ Base & & Observation Point No. \\
\hline & & $3^{n}$ Base & & & $3^{n \text { Base }}$ & & Necessary Data \\
\hline & & Mixed & & & Mixed & & Additional Observation Point No. \\
\hline & \multirow{3}{*}{ Count } & Strike & & \multirow{3}{*}{ Count } & Mixed & & \\
\hline & & Ball & & & Ball & & \\
\hline & & Out & & & Out & & \\
\hline & \multirow{3}{*}{ Hitter } & ID & & \multirow{3}{*}{ Hitter } & ID & & \\
\hline & & Times at Bat & & & Times at Bat & & \\
\hline & & No. of Throw & & & \begin{tabular}{|l|} 
No. of Throw \\
\end{tabular} & & \\
\hline & \multirow{3}{*}{ Pitcher } & ID & & \multirow{3}{*}{ Pitcher } & ID & & \\
\hline & & No. of Throw & & & No. of Throw & & \\
\hline & & $\begin{array}{l}\text { No. of Throw } \\
\text { Inning }\end{array}$ & & & $\begin{array}{l}\text { No. of Throw } \\
\text { Inning }\end{array}$ & & \\
\hline & Previous Contex & & $O P$ & Has Relation & & & \\
\hline & & & Or & Has Previous $\mathrm{C}$ & & & \\
\hline
\end{tabular}

Fig. 7. Model for Relation between Context and Observation Point 
Context elements are grouped in ontology structure for the contexts to be recognized according to the data type property. Inning, runner, count, scoring margin, etc. are groups in high level. Inning has inning number and first/second half as lower grouping elements. Count has ball, strike, and out. The ontology structure has been modeled in the way contexts can be specifically represented with these grouping elements. The context formulated with a series of events can be represented since the context class in this study contains the relation with the previous context. The change in context along with the progress of events can be expressed as the sequence is controlled with 'previous context number'. Server can identify the context change over time according to the representation defined in the ontology model. Variety of the observation points have been derived since the context change over time can be expressed and the server is able to recognize the context change through the model represented in ontology.

\section{Implementation of Context-Based Information Service for Baseball Game}

The information service system for providing observation points and relevant information during the baseball play has been realized in order to assess the usability of the context based information agent. The system has been implemented in the server/client structure based on internet as shown on Fig. 8. The real time information on a game in progress and the video stream of the game are supplied to the server at the ball park. The situation related observation points are derived and indexed by the agent according to the level of relevance. The observation points and related data are stored along with the concurrent video image, and these are supplied to the client on request. iPhone has been used as the client device for mobile service.

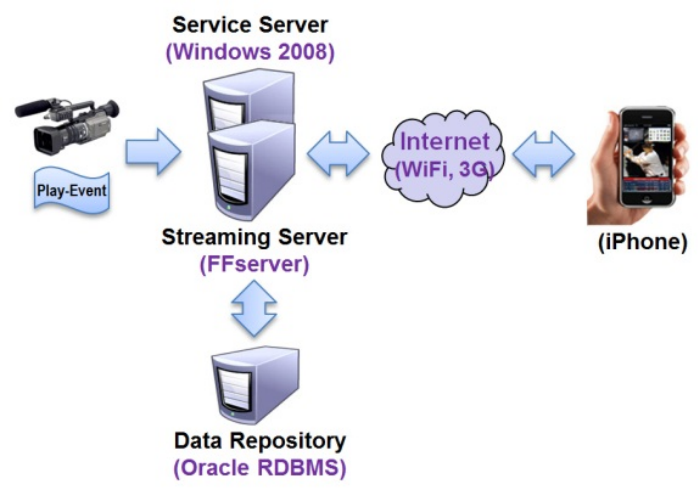

Fig. 8. Platform for the Information Service System

A client App has been developed, which can supply video stream of the ball game, real time observation points, game progress information, player information, etc through the user interface as shown on Fig. 9. The client App regularly requests the server for the observation points along with the timing of the video streaming of a 

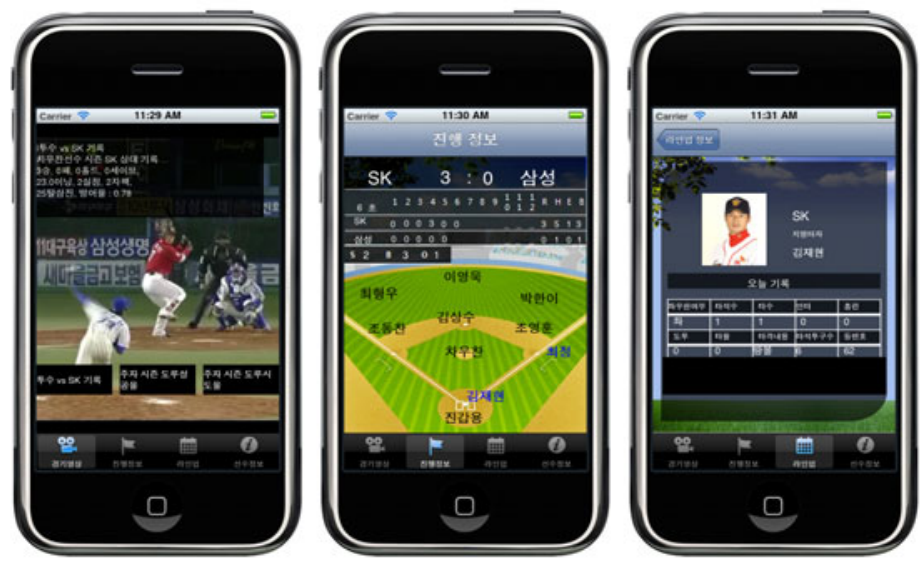

Fig. 9. User Interfaces for Baseball Information Service

game. The game situation information and the player information are supplied only on demand from user. Only top three of the suggested observation points are displayed at the bottom considering screen size of smart phone. Once any of the observation points is selected by the user, the relevant data are displayed on the top of the screen. The information is overlaid on the video streaming of the ball game. The system has been applied to the baseball games of Korean Series 2010. The system was appreciated that understanding over the progress of the match had been enhanced and the interest and immersion in the game had been increased by the information service.

\section{Discussion}

In this work, an intelligent information service system for baseball has been implemented as a context based information service agent. Recording interface for the acquisition of the progress information of ball game and for the context modeling has also been designed. An ontology model has been structured for being aware of contextual change over time. The ontology is also designed for parsing data to knowledge and extracting observation points. The observation points which is the representation of groups of the information appealing to the user at a certain context are listed in order according to the relative importance and displayed on the mobile device as shown in Fig. 9.

The implemented system satisfactorily offered information at every change of the situation, i.e. ball count, out count, inning, score and player while the system was applied to the real match of Korean Baseball League. In an actual situation when runners on the first and third base at the out count one for instance, the system suggested the possibility of hit, base steal, and double play. Once the possibility of double play was selected, the record of the batter on the double play against the pitcher during the season and with some other condition was displayed. As the observation points were listed first for the user to select and the record information in detail was supplied only when any of the points was selected, the service was appreciated non-invasive and timely. The scope of this agent can be expanded to other domains like tourism, e-learning, and e-health since the ontology can be modified and expanded as required. 


\section{References}

1. Dey, A.K., Abowd, G.D., Salber, D.: Conceptual Framework and a Toolkit for Supporting the Rigid Prototyping of Context-Aware Applications. Human-Computer Interaction 16, 97-106 (2001)

2. Kim, J., Hahn, D., Lim, J.: Intelligent Location Guide System based on Context Awareness. In: Proceeding of Conference of Korean Internet Information Society, pp. 683-686 (2010) (in Korean)

3. Ganzha, M., Paprzycki, M., Gawinecki, M., Szymczak, M., Frackowiak, G., Bădică, C., Popescu, E., Park, M.-W.: Adaptive Information Provisioning in an Agent-Based Virtual Organization - Preliminary Considerations. In: Negru, V., et al. (eds.) Proceedings of the SYNASC Conference, pp. 235-241. IEEE CS Press, Los Alamitos (2007)

4. Chang, H., Roh, J., Cho, S.: Context based Use Control Model for Mobile Device. Society of Information Science Journal 14, 63-70 (2008)

5. Dey, A.K., Abowd, G.D.: Towards a Better Understanding of Context-Awareness. In: CHI 2000 Workshop on The What, Who, Where, When, and How of Context-Awareness (2000)

6. Rhodes, B., Maes, P.: Just-in-time information retrieval agents. IBM Systems Journal Special Issue on the MIT Media Laboratory, 39(3and4), 685-704 (2000) 